\title{
Vincent Meyer, Communication organisationnelle et prise en charge du handicap mental
}

Les Études Hospitalières Éditions, Bordeaux, 2005, 206 pages

\section{Elizabeth Gardère}

\section{OpenEdition}

\section{Journals}

Édition électronique

URL : http://journals.openedition.org/communicationorganisation/407

DOI : 10.4000/communicationorganisation.407

ISSN : 1775-3546

\section{Éditeur}

Presses universitaires de Bordeaux

Édition imprimée

Date de publication : 1 décembre 2007

Pagination : 241-243

ISSN : $1168-5549$

Référence électronique

Elizabeth Gardère, «Vincent Meyer, Communication organisationnelle et prise en charge du handicap mental », Communication et organisation [En ligne], 32 | 2007, document 18, mis en ligne le 01

décembre 2007, consulté le 21 septembre 2020. URL : http://journals.openedition.org/

communicationorganisation/407 ; DOI : https://doi.org/10.4000/communicationorganisation.407 


\section{Vincent Meyer, Communication organisationnelle et prise en charge du handicap mental}

Les Etudes Hospitalières Editions, Bordeaux, 2005, 206 pages.

Le regard critique et avisé que porte Vincent Meyer sur le milieu social et médico-social éclaire les figures communicationnelles qui entourent la prise en charge du handicap mental. C'est à la lumière de son parcours de praticien en tant que travailleur social en milieu institutionnel, puis d'enseignant-chercheur en tant que professeur à l'université de Paul Verlaine-Metz qu'il nous livre son retour d'expériences. Son constat révèle les pratiques professionnelles et y associe les outils communicationnels adéquats. Le choix de la méthodologie de la recherche-action, soutenu par l'évaluation ethnographique, ouvre sur la valeur de l'information en milieu institutionnel en termes d'intelligibilité, de qualité et d'évaluation.

L'ouvrage décline la problématique en trois temps qui ponctuent le parcours du lecteur. Après avoir pris connaissance des protagonistes de la communication en milieu institutionnel, la représentation du handicap est examinée au prisme des interactions et du lien entre la communication interpersonnelle et la circulation de l'information. Ce cheminement réflexif qui considère les rituels d'interaction, conduit à s'interroger sur les compétences communicationnelles et l'évaluation des professionnels en charge le handicap mental. L'expertise participative est convoquée dans le cadre d'une analyse qui s'appuie sur les enseignements du partage d'expériences (parfois à défaut de la capitalisation des savoirs). Cela tant sur le plan des connaissances constituées que sur celui des échanges informels. La description ethnographique, socle du corpus, et la démarche participative de connaissance et d'étude de la réalité professionnelle, s'inscrivent dans le prolongement des recherches en sociologie du travail entreprises par l'auteur et positionnent l'ancrage théorique de ce livre.

Dans cette perspective d'analyse des pratiques et discours des professionnels de la santé mentale, les établissements sociaux et médico-sociaux offrent à la recherche-action un éventail de contextes, de situations d'observation participante et de conduites collectives qui concourent à l'évaluation des pratiques. Vincent Meyer s'en empare et tissent des formes organisationnelles et communicationnelles dans un espace institutionnel en évolution. L'ambition est de donner lecture 


\section{Bibliographie}

des méandres des processus de socialisation avant d'entreprendre l'organisation des processus de communication des conduites socioéducatives où l'altérité occupe une place centrale. Cette préoccupation est éminemment d'actualité dans un espace public en prise avec le repliement communautaire, la montée de l'individualisme et l'exclusion qui questionnent le statut et la place du Sujet. L'auteur relate ici les conditions de prise en charge des personnes handicapées mentales avec pragmatisme et humanité, et va au-delà d' "une logique d'action des individus, mais bien dans une logique de champs de production chère à Pierre Bourdieu ». (Meyer, 2005: 10-11) en convoquant les couples techniques, pragmatiques et les représentations pour donner corps à la communication.

L'approche communicationnelle de la dynamique de socialisation mise en exergue par Vincent Meyer témoigne de l'importance des représentations dans les modes d'appropriation et de transmission de l'information. En effet, ces représentations influencent notre rapport au monde, aux autres, à soi. L'enjeu de la seconde partie est de se distancier des représentations que donne à voir l'institution pour percevoir les non-dits, les usages et les logiques sous-jacentes qui conditionnent les conduites éducatives. L'intention est de les évaluer et de proposer des solutions pour passer des conduites aux pratiques.

Malgré l'importance que revêt un projet collectif pour apporter sa pierre à l'édifice dans la prise en charge du handicap mental, force est de constater que la démarche des professionnels de la santé mentale observés dans le cadre de ce travail, semble être plus individuelle qu'une œuvre commune. Elle s'apparente davantage à la collecte d'informations éparses issues de manuels, procédures et règlements. Cette dimension hétérogène s'éloigne du projet fédérateur qui participe à l'apprentissage collectif. La thématique sur laquelle repose le système institutionnel de la prise en charge du handicap s'articule surtout autour des relations interpersonnelles, expression d'un modèle plus ou moins codé, réglé, normé par une phraséologie qui tend, dans l'idéal, vers «des effets de changement concernant la maladie ou l'inadaptation" (Meyer, 2005: 8), mais de manière plus factuelle vers des objectifs.

Par ailleurs, le développement des technologies de l'information et de la communication n'est pas un fait avéré dans le milieu institutionnel du handicap, même si la psychologie cognitive s'engage dans cette voie plus rapidement que d'autres. Le passage du projet à l'action suppose une démarche d'appropriation des outils d'informatisation. Cette étape à franchir dans le métier d'éducateur, entre autres, rompt avec la primauté des relations interpersonnelles et freine l'intérêt porté 
aux TIC. Leur usage assurerait pourtant la promotion des politiques publiques en faveur des personnes handicapées, de leur famille et des structures d'encadrement. L'introduction de l'e-communication et de l'e-travail social, bien que lente, offre des perspectives pour évaluer et améliorer les «formes de coordination interne et externe des prises en charge institutionnelles lorsqu'elles sont dépendantes de machines ou d'artefacts et sur l'absence d'une mémoire collective instituée» (Meyer, 2005 : 191).

Conçu comme un retour d'expériences à portée théorique, ce livre témoigne des stratégies langagières des travailleurs sociaux à verbaliser leurs pratiques et à transmettre leur message éducatif. D'après une méthode articulée autour de scenarii fictifs ou réels, ils relatent leur quotidien à la croisée de la vie institutionnelle d'une personne handicapée mentale, de sa représentation, de l'imaginaire collectif qui l'entoure et du rôle du personnel médical qui côtoie une dépendance socialement avérée, individuellement redoutée. La portée communicationnelle de cette analyse met l'accent sur les modes opératoires d'accompagnement et d'apprentissage comme leviers de la relation socio-éducative confrontée à l'efficacité de l'acte professionnel.

Le lecteur retiendra que les conflits d'organisation, le sens des interactions et les actions éducatives en milieu médico-social sont tiraillés entre les intentions, représentations et pratiques sociales. Il comprendra pourquoi il n'est pas de réponse tranchée ni sur le passage de l'expérience à la connaissance, ni sur la transmission de l'information et sa déclinaison en savoirs professionnels. Problématiques qui restent au cœur des recherches de Vincent Meyer. Cet ouvrage n'est pas uniquement destiné à ceux concernés par la thématique de la santé. Il touche plus largement tous ceux qui, confrontés à la recherche-action, questionnent leur terrain et la valeur de la communication interpersonnelle sur et dans le social.

Elizabeth Gardère 